\title{
ARTICLE OPEN The formation of perovskite multiple quantum well structures for high performance light-emitting diodes
}

\author{
Yan Sun ${ }^{1}$, Li Zhang ${ }^{1}$, Nana Wang $\mathbb{D}^{1}$, Shuting Zhang ${ }^{1}$, Yu Cao ${ }^{1}$, Yanfeng Miao ${ }^{1}$, Mengmeng Xu ${ }^{1}$, Hao Zhang ${ }^{1}, \mathrm{Hai}^{\mathrm{Li}} \mathbb{D}^{1}, \mathrm{Channg}^{\mathrm{Y}}{ }^{1}$,
} Jianpu Wang (iD) and Wei Huang ${ }^{1,2}$

Recent works showed that high efficient perovskite light-emitting diodes can be achieved from solution-processed, self-organized multiple quantum wells (MQWs) with an energy cascade. We investigate how the mixing of QWs with different band gaps can affect the perovskite LED device performance. We find that the annealing process can significantly affect the constitution of the MQWs films, where the dominant phase can evolve from large band gap QWs to small band gap QWs. The optimal constitution for LED application lies in a transition point of small- $n$ QWs dominant phase to large- $n$ QWs dominant phase, when the MQW film presents highest photoluminescence while still remains uniform film morphology.

npj Flexible Electronics (2018)2:12; doi:10.1038/s41528-018-0026-0

\section{INTRODUCTION}

Solution-processable organic-inorganic hybrid perovskites have been of interest due to their application in flexible, high efficiency, and low cost solar cells and light-emitting diodes (LEDs). ${ }^{1-9}$ For perovskite LED (PeLED) applications, the most important issue is the nonradiative recombination caused by poor film uniformity or defects. ${ }^{3}$ Recent work has demonstrated that high efficiency and stable PeLEDs can be achieved based on self-organized multiple quantum wells (MQWs) structure with an energy cascade. ${ }^{4,6}$ The MQW perovskite is a mix of layered perovskites with different band gaps resulting from various layer numbers $(n)$ of lead halide (Fig. 1). It can combine advantages of both two dimensional (2D) perovskites and three dimensional (3D) perovskites. ${ }^{10,11}$ Very smooth film morphology, similar as pure 2D perovskite film, can be formed from the MQW perovskites. $^{6,12}$ More importantly, it can have high photoluminescence quantum efficiencies (PLQEs) due to the fast energy transfer from large band gap QWs to small band gap QWs where efficient emission occurs, which effectively avoid the fast excitonphonon quenching in pure 2D perovskites. ${ }^{6,13-15}$ Clearly, the constitution of the MQW is important to determine the device performance of PeLEDs, while it has not been studied. Here we investigate how the mixing of QWs with different band gaps will affect the PeLED device performance. We find that the annealing process can significantly affect the constitution of the MQWs which evolve from a dominant phase of small- $n$ QWs to large- $n$ QWs. The optimal constitution for LED application lies in a transition point of small- $n$ QWs dominant phase to large- $n$ QWs dominant phase, where the MQW film presents highest photoluminescence $(\mathrm{PL})$ while still remains uniform film morphology. This work can provide a guideline for the further enhancement of the performance of PeLEDs.

\section{RESULTS}

A precursor solution is prepared with 1-naphthylmethylamine iodide (NMAl), formamidinium iodide (FAl), and $\mathrm{Pbl}_{2}$ dissolved in $\mathrm{N}, \mathrm{N}$-dimethylformamide (DMF) with a molar ratio of 2:1:2. The MQW film (refer as $\mathrm{NFPI}_{7}$ ) is formed by spin-coating the precursor solution onto substrates of $\mathrm{ZnO} / \mathrm{PEIE}$-coated ITO glass followed by annealing at $100^{\circ} \mathrm{C}$, which is same as our LED device fabrication process. $^{6}$ We note that the MQW formation process is slightly different when different underneath layer (e.g., bare glass substrate) is used, but the overall observation is consistent (Supplementary Figure 1).

Figure $2 \mathrm{a}$ shows the absorption spectra of $\mathrm{NFPl}_{7}$ film annealed at various annealing time. The as-spun film shows a weak absorption peak at $567 \mathrm{~nm}$ which is due to the exciton absorption of $n=2 \mathrm{QWs}^{6,16}$ indicating a small number of $n=2 \mathrm{QWs}$ have already been formed during the spin-coating process. Upon annealing, the absorption from the $n=2$ QWs quickly increases, reaching maximum in $10 \mathrm{~min}$ and gradually decreases with extended annealing time. The absorption peak at $632 \mathrm{~nm}$ can also be observed with the $10 \mathrm{~min}$ annealed film, which suggests the growing of $n=3$ QWs during the annealing process., ${ }^{6} 13$ The long tail of the absorption spectra till $\sim 785 \mathrm{~nm}$, from the absorption of large-n QWs, grows over the annealing time. The 60 min annealed film almost shows no distinguishable absorption peaks from $n=2$ or $3 \mathrm{QWs}$. The absorption measurement result suggests that the annealing process facilitates to form both small$n$ and large- $n$ QWs at the early stage, followed by decomposition of small-n QWs and formation of more large- $n$ QWs upon extended annealing time. We speculate that after decomposition of small- $n$ QWs, the long chain amine $\mathrm{NMA}^{+}$can modify surface defects and fill in the gaps between perovskite crystals.

This formation process can be confirmed by X-ray diffraction (XRD) measurement (Fig. 2b). The as-spun film shows a very weak

\footnotetext{
${ }^{1}$ Key Laboratory of Flexible Electronics (KLOFE) \& Institute of Advanced Materials (IAM), Jiangsu National Synergetic Innovation Center for Advanced Materials (SICAM), Nanjing Tech University (NanjingTech), 30 South Puzhu Road, Nanjing 211816, China and ${ }^{2}$ Shaanxi Institute of Flexible Electronics (SIFE), Northwestern Polytechnical University (NPU), 127 West Youyi Road, Xi'an 710072 Shaanxi, China

Correspondence: Jianpu Wang (iamjpwang@njtech.edu.cn) or Wei Huang (iamwhuang@nwpu.edu.cn)
}

Received: 3 May 2017 Revised: 13 March 2018 Accepted: 29 March 2018

Published online: 23 April 2018 

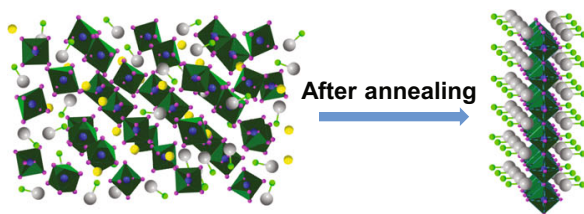

$n=1$

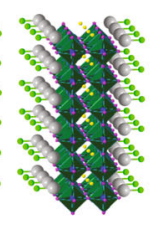

$\mathrm{n}=\mathbf{2}$

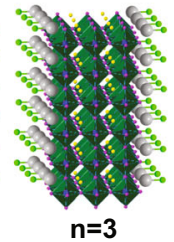

$\mathrm{NMA}^{+}$

Fig. 1 Schematic representation of the evolution of perovskite MQW constitutions upon annealing. The resulted MQW perovskite is a mix of layered perovskites with different layer numbers $(n)$ of lead halide
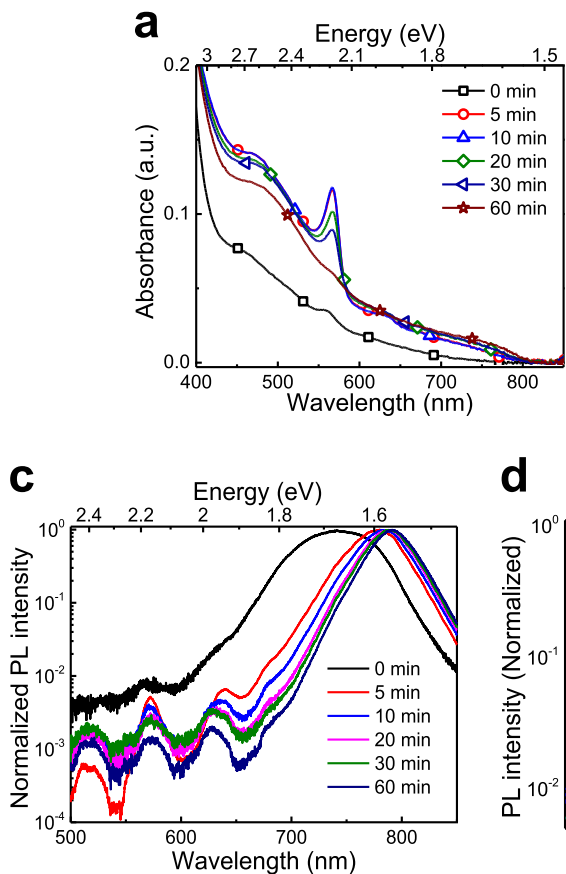

d

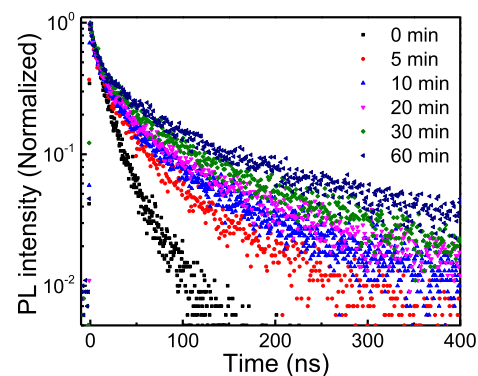

b

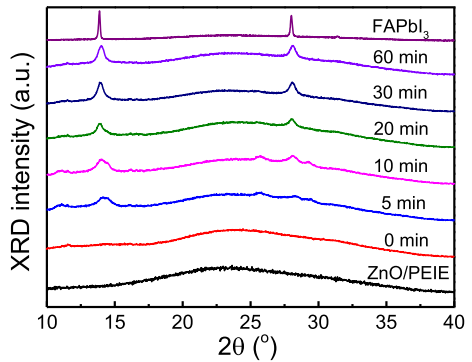

e

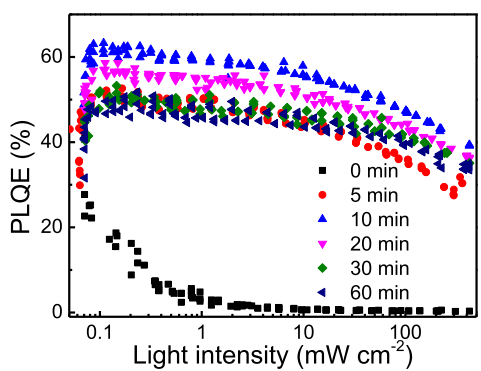

Fig. 2 Perovskite MQW films deposited on PEIE modified ZnO layers. a Absorption spectra of $\mathrm{NFPI}_{7}$ films upon various annealing time. $\mathbf{b}$ XRD data of $\mathrm{FAPbl}_{3}, \mathrm{ZnO} / \mathrm{PEIE}$, and NFPI films upon various annealing time. c PL spectra of $\mathrm{NFPI}_{7}$ films excited by a $445 \mathrm{~nm}$ laser. $\mathbf{d}$ Time-resolved photoluminance of $\mathrm{NFPI}_{7}$ films annealed for various time. e Excitation-intensity-dependent PLQE of MQW perovskite films upon various annealing time. The most efficient emission is from the $10 \mathrm{~min}$ film which is around $60 \%$

peak at $11.5^{\circ}$ and $16.2^{\circ}$, and it becomes more obvious with the $5 \mathrm{~min}$ annealed film. Additionally, the $5 \mathrm{~min}$ annealed film shows several new peaks at $11.0^{\circ}, 13.9^{\circ}, 14.5^{\circ}, 25.7^{\circ}, 28.1^{\circ}$, and $29.3^{\circ}$. The peaks at $13.9^{\circ}$ and $28.1^{\circ}$ are consistent with the diffraction from the a-phase of $3 \mathrm{D} \mathrm{FAPbl}{ }_{3}{ }^{7,17,18}$ These two peaks significantly increase over the annealing time, suggesting the annealing process helps to form more large-n QWs. The other peaks, $11.0^{\circ}$, $14.5^{\circ}, 25.7^{\circ}$, and $29.3^{\circ}$ can be attributed to small- $n$ layered perovskites, ${ }^{19-21}$ which decrease as the annealing time exceeding $10 \mathrm{~min}$. Therefore, the XRD result suggests that the formation of different $n$ QWs is a competing process in the MQW film. The small- $n$ QWs are quickly formed upon annealing, and the formation reaches to peak value in $\sim 10 \mathrm{~min}$. While the large- $n$ QWs keep growing upon the annealing process with the decomposition of small- $n$ QWs.

We find that the change of constitution of the MQW films by the annealing process can profoundly affect the film optical properties. As shown in Fig. 2c, the PL spectrum of $5 \mathrm{~min}$ film shows a strong main peak from large- $n$ QWs and several weak emission peaks at 516,574, 635, and a shoulder at $678 \mathrm{~nm}$, corresponding to the emission from $n=1,2,3$, and 4 QWs respectively. $6,13,16,20,22$ The $P L$ from different $n$ perovskites can be more clearly presented at low temperature (Supplementary Figure
2), demonstrating that the perovskite film has a cascade energy structure from different- $n$ of layered perovskites. The PL at different emission peaks have markedly different lifetimes (Supplementary Figure 3). The emissions at 574 and $635 \mathrm{~nm}$ show extremely short lifetimes, and the PL lifetime increases as the emission photon energy decreases, indicating a fast and efficient cascade energy transfer from the small- $n$ QWs to large- $n$ QWs. With longer annealing time, the emission peaks from these small$n$ QWs decreases and the main emission peak is red shifted. The $\mathrm{PL}$ emission of $60 \mathrm{~min}$ annealed film peaks at $787 \mathrm{~nm}$, and $\sim 10 \mathrm{~nm}$ blue-shifted compared to that of $3 \mathrm{D} \mathrm{FAPbl}_{3}$ perovskite., 17 Therefore, the PL measurement is consistent with the scenario that the process of increasing of large- $n$ QWs with the decomposition of small- $n$ QWs upon long time annealing. Furthermore, transient $\mathrm{PL}$ measurement shows that the $\mathrm{PL}$ lifetimes at the main emission peaks increase over the annealing time (Fig. 2d), consistent with the PL contribution from the larger QWs with less confinement effect. More importantly, the lightintensity-dependent PLQE measurement shows that the most efficient emission is from the $10 \mathrm{~min}$ film (Fig. 2e), indicating the importance of MQW constitution for the emission efficiency.

In addition, we find that the constitution of the MQW films can also affect the film morphology which is important for fabricating 
a
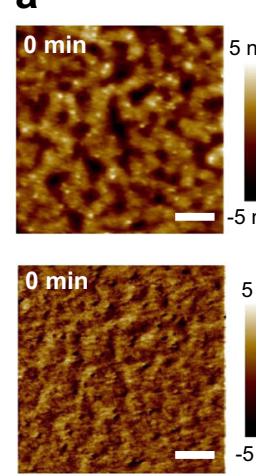

b

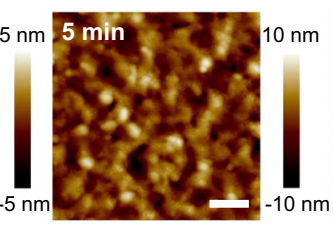

c

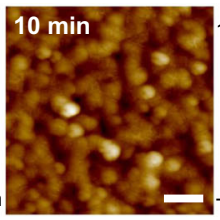

d

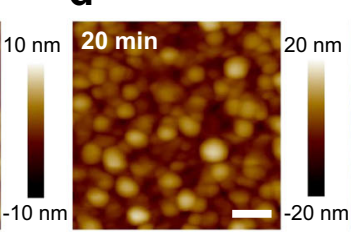

e

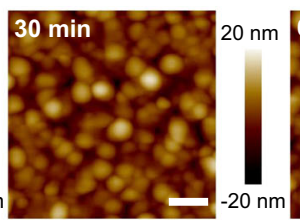

f

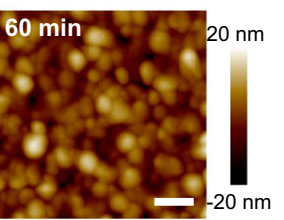

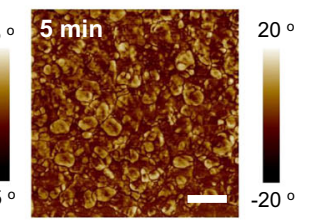
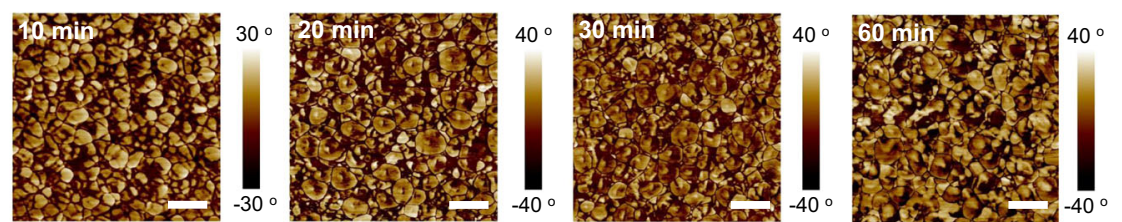

Fig. 3 Atomic force microscopy images of the MQW perovskite films upon various annealing time (scale bar, $200 \mathrm{~nm}$ ). The height and phase graphs show the film roughness increasing and grain growth along with annealing time, respectively. a 0 min. b 5 min. c 10 min. $\mathbf{d} 20$ min. e $30 \mathrm{~min}$. f $60 \mathrm{~min}$

\begin{tabular}{|lllll|}
\hline \multicolumn{5}{|l}{$\begin{array}{l}\text { Table 1. } \\
\text { annealing time and the corresponding LED device performance }\end{array}$} \\
\hline $\begin{array}{l}\text { Annealing time } \\
(\mathrm{min})\end{array}$ & $\begin{array}{l}R_{\mathrm{rms}} \\
(\mathrm{nm})\end{array}$ & $\begin{array}{l}\text { Peak PLQE } \\
(\%)\end{array}$ & $\begin{array}{l}\text { Peak EQE } \\
(\%)\end{array}$ & $\begin{array}{l}\text { EL peak } \\
(\mathrm{nm})\end{array}$ \\
\hline 0 & 1.4 & 25 & - & - \\
5 & 2.7 & 50 & 4.2 & 768 \\
10 & 3.5 & 60 & 8.6 & 785 \\
20 & 4.1 & 56 & 7.7 & 785 \\
30 & 4.3 & 50 & 5.1 & 787 \\
60 & 4.8 & 47 & 2.8 & 787 \\
\hline
\end{tabular}

LED devices. ${ }^{1,3}$ As shown in Fig. 3, the film roughness shows a tendency of increasing during the annealing process (Table 1). When take the phase diagram into account, shown in the bottom of height graph, grain growth along with annealing time can be clearly observed. The as-spun film is almost featureless. After $5 \mathrm{~min}$ annealing, the phase diagram shows that some grains with $\sim 100 \mathrm{~nm}$ diameter are emerging. More grains appear with longer annealing time. We assume these grains growing over annealing time are large- $n$ QW domains, since the growth process is consistent with what we have learned from the spectroscopic measurement. This assumption can be verified by cross section high-resolution transmission electron microscopy (HRTEM) measurement of a $10 \mathrm{~min}$ annealed film. Figure 4a shows that the bright region on the dark field of HRTEM image, which is from large- $n$ QWs, ${ }^{6}$ mainly locates at the top of the perovskite MQW films.

So far, we have investigated the evolution of perovskite MQW constitutions upon annealing time. We then proceed to fabricate devices based on those different MQW films. Figure 4a shows that the device structure is indium tin oxide (ITO)/polyethylenimine ethoxylated (PEIE)-modified zinc oxide $(\mathrm{ZnO}, \sim 20 \mathrm{~nm}) /$ perovskite MQWs $\quad(\sim 30 \mathrm{~nm}) /$ poly(9,9-dioctyl-fluorene-co-N-(4-butylphenyl) diphenylamine) (TFB, $\sim 40 \mathrm{~nm}) /$ molybdenum oxide $\left(\mathrm{MoO}_{\mathrm{x}}\right.$ $\sim 7 \mathrm{~nm}) /$ gold $(\mathrm{Au}, \sim 60 \mathrm{~nm})$. Figure $4 \mathrm{~b}$ shows the current density-voltage-radiance $(J-V-R)$ characteristics of the electroluminescence (EL) devices based on the MQW films with different annealing time. We note there is no measureable EL performance with the as-spun $\mathrm{NFPI}_{7}$ film. As annealing time increases, the turnon voltage shows a slight decrease likely due to the reduced charge injection barrier. Figure $4 c$ shows the EL device external quantum efficiencies (EQEs) are significantly affected by the annealing time, suggesting the importance of the constitution of MQWs for LED applications. The best performing LED device is from the $10 \mathrm{~min}$ annealed film, reaching $8.6 \%$ at $2.45 \mathrm{~V}$ with a current density of $25.0 \mathrm{~mA} \mathrm{~cm}^{-2}$, and a high radiance of $10.8 \mathrm{~W}$ $\mathrm{sr}^{-1} \mathrm{~m}^{-2}$. We note the EL EQE performance (Table 1) of different annealed film is consistent with the PLQE measurements. In addition, the fact that the MQW film becomes more rough over the annealing time can also deteriorate the EL device performance. $^{1,6,23}$

Figure $4 d$ shows the EL emission peaks red shift from 768 to $785 \mathrm{~nm}$ as the annealing time from 5 to $10 \mathrm{~min}$, and remain almost constant over $10 \mathrm{~min}$. In EL device operation, electrons and holes are injected into the perovskite layer, accumulated and recombined at the large- $n$ QWs where have the smallest energy gap. This is consistent with that the weak emissions at higher energies shown in the PL measurements are absent in the EL spectra. The change of EL emission peak over annealing time suggests that the $n$-number of large-n QW increase during initial 10 min annealing, and it almost remains constant after $10 \mathrm{~min}$. Together with above absorption and PL measurement, we can infer that there forms more and more QWs with large- $n$ number when the annealing time over $10 \mathrm{~min}$.

\section{DISCUSSION}

We have investigated the influence of thermal annealing on the morphology and optical properties of self-organized perovskites MQWs. We find upon annealing, the MQW constitution evolves from a small- $n$ QWs dominant phase to large- $n$ QWs dominant phase. The optimal constitution for LED application lies in the transition point of small- $n$ QWs dominant phase to large- $n$ QWs dominant phase. In this constitution, the MQW perovskite film presents highest PLQE while still remain uniform film morphology. We believe this finding paves the way for further improve the device performance of MQW based PeLEDs.

In group III-V semiconductor based MQW LED devices, precise control of the quantum well configuration is required to achieve good electrical and optical properties. We show that the configuration of self-organized perovskites MQWs can be easily controlled upon low-temperature thermal annealing process, resulting in high-efficiency LED devices. This study further indicates the MQW perovskites are promising for realizing high performance, large area, and low cost LED devices.

\section{METHODS}

Film and device fabrication

The $\mathrm{ZnO}$ nanocrystals and $\mathrm{NFPI}_{7}$ solution were prepared as previous report. ${ }^{6}$ The $\mathrm{NFPl}_{7}$ films were deposited at $4000 \mathrm{rpm}$ for $45 \mathrm{~s}$ on quartz and $\mathrm{ZnO/PEIE-coated} \mathrm{ITO} \mathrm{glass} \mathrm{substrates,} \mathrm{respectively.} \mathrm{The} \mathrm{resulting} \mathrm{films} \mathrm{were}$ 
a
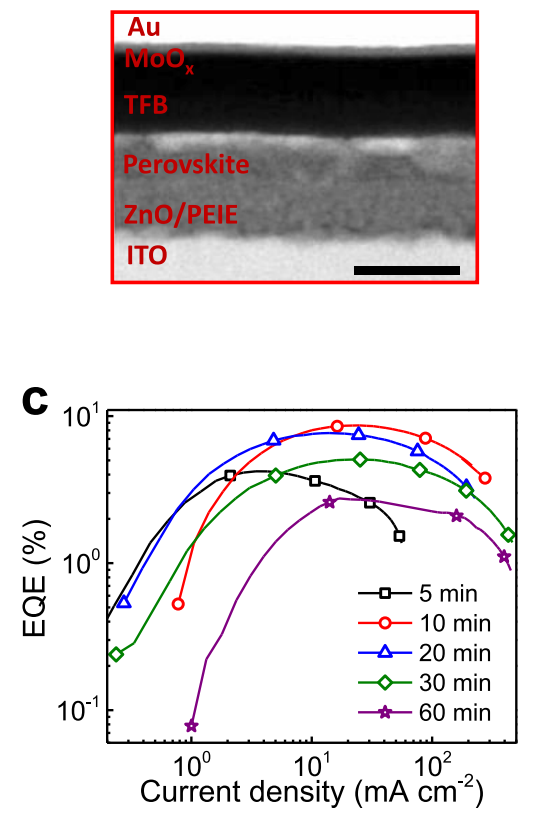

b

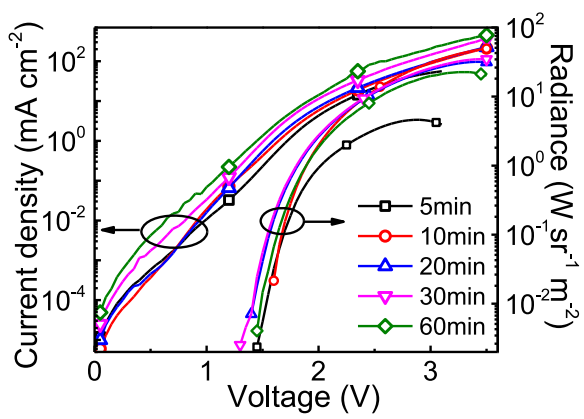

d

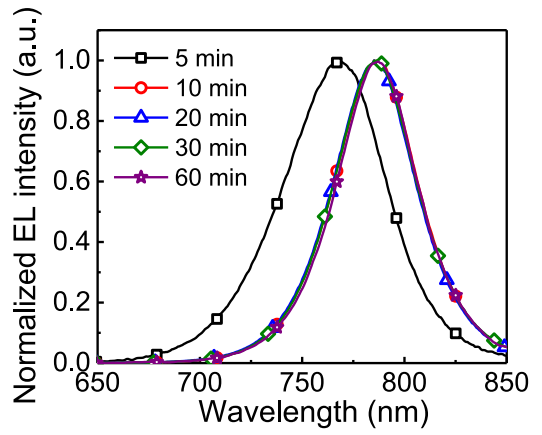

Fig. 4 Device characteristics of the perovskite MQW LEDs. a A cross-sectional HRTEM image for MQW LED with a 10 min annealed perovskite film (scale bar, $50 \mathrm{~nm}$ ). b Dependence of current density and radiance on the driving voltage. c EQE versus current density. d EL spectra showing the emission peaks red shift as the annealing time increasing

then annealed at $100^{\circ} \mathrm{C} .1 .2 \times 1.2 \mathrm{~cm}^{2}$ ITO-coated glass with $15 \Omega / \mathrm{sq}$ was selected as substrate for device fabrication. The deposition method of hole-transport layer, TFB and top electrodes can be found elsewhere. ${ }^{6}$

\section{Characterization}

UV-vis absorbance of the perovskite films were measured using an Agilent Cary 5000 UV-vis spectrophotometer with an integrating sphere. The PL spectra were record by Andor spectrograph (Shamrock-303i-B) with the iDus490A-1.7 CCD. The morphology of the perovskite layers were characterized by using atomic force microscopy (Bruker, Dimension Icon SPM). The time resolved fluorescence spectra were obtained by using an Edinburgh Instruments (FLS920) spectrometer with a $445 \mathrm{~nm}$ laser as excitation light source (excitation intensity, $\sim 0.2 \mu \mathrm{J} \mathrm{cm}^{-2}$ ). XRD data were obtained by a Philips X-ray diffractometer with CuKa radiation. The HRTEM images were collected on a Tecnai G2 F20 microscope operated at $200 \mathrm{keV}$. The PLQEs of perovskite films were measured by a three-step technique which combined laser, optical fiber, spectrometer, and integrating sphere. ${ }^{24}$ For LED device characterizations, a Keithley 2400 source meter was used for J-V measurement and a fiber integration sphere (FOIS-1) couple with a QE-6500 spectrometer was used for the light output measurement.

Data availability

The data that support the finding of this study are available from the corresponding author upon reasonable request.

\section{ACKNOWLEDGEMENTS}

This work is financially supported by the Major Research Plan of the National Natural Science Foundation of China (91733302), the National Basic Research Program of China-Fundamental Studies of Perovskite Solar Cells (2015CB932200), National Key Research and Development Program of China (2017YFB0404501), the Natural Science Foundation of Jiangsu Province, China (BK20140952, BK20150043, BK20170991), the National Natural Science Foundation of China $(11474164,61634001,51703094)$, the Joint Research Program between China and European Union (2016YFE0112000), the National Science Fund for Distinguished Young Scholars (61725502), the Synergetic Innovation Center for Organic Electronics and Information Displays. We thank Y. Liu and J. Li for the assistance of the TCSPC measurement.

\section{AUTHOR CONTRIBUTIONS}

J.W. had the idea for and designed the experiments. J.W. and W.H. supervised the work. Y.S. and M.X. conducted the optical measurements. L.Z. and S.Z. carried out the device fabrication and characterizations. C.Y. and Y.C. synthesized the NMAI. H.Z. and H.L. measured AFM. Y.M. carried out HRTEM characterizations. J.W., N.W., and W.H. participated in data analysis and wrote the manuscript. All authors discussed the results and commented on the manuscript.

\section{ADDITIONAL INFORMATION}

Supplementary information accompanies the paper on the npj Flexible Electronics website (https://doi.org/10.1038/s41528-018-0026-0).

Competing interests: The authors declare no competing interests.

Publisher's note: Springer Nature remains neutral with regard to jurisdictional claims in published maps and institutional affiliations.

\section{REFERENCES}

1. Tan, Z. K. et al. Bright light-emitting diodes based on organometal halide perovskite. Nat. Nanotech. 9, 687-692 (2014).

2. Saliba, M. et al. Incorporation of rubidium cations into perovskite solar cells improves photovoltaic performance. Science 354, 206-209 (2016).

3. Wang, J. et al. Interfacial control toward efficient and low-voltage perovskite light-emitting diodes. Adv. Mater. 27, 2311-2316 (2015).

4. Yuan, M. et al. Perovskite energy funnels for efficient light-emitting diodes. Nat. Nanotech. 11, 872-877 (2016).

5. Tsai, H. et al. High-efficiency two-dimensional ruddlesden-popper perovskite solar cells. Nature 536, 312-316 (2016).

6. Wang, N. et al. Perovskite light-emitting diodes based on solution-processed selforganized multiple quantum wells. Nat. Photonics 10, 699-704 (2016).

7. Yang, W. S. et al. High performance photovoltaic perovskite layers fabricated through intramolecular exchange. Science 348, 1234-1237 (2015).

8. Wang, Y. et al. High-efficiency flexible solar cells based on organometal halide perovskites. Adv. Mater. 28, 4532-4540 (2016).

9. Wang, N., Si, J., Jin, Y., Wang, J. \& Huang, W. Solution-processed organic-inorganic hybrid perovskites: a class of dream materials beyond photovoltaic applications. Acta Chim. Sin. 73, 171-178 (2015).

10. Mitzi, D. B. Organic-inorganic electronics. IBM J. Res. Dev. 45, 29-45 (2001). 
11. Saparov, B. \& Mitzi, D. B. Organic-inorganic perovskites: structural versatility for functional materials design. Chem. Rev. 116, 4558-4596 (2016).

12. Zhang, S. et al. Efficient red perovskite light-emitting diodes based on solutionprocessed multiple quantum wells. Adv. Mater. 29, 1606600 (2017).

13. Hong, X., Ishihara, T. \& Nurmikko, A. V. Dielectric confinement effect on excitons in $\mathrm{Pbl}_{4}$-based layered semiconductors. Phys. Rev. B 45, 6961-6964 (1992).

14. Gauthron, K. et al. Optical spectroscopy of two-dimensional layered $\left(\mathrm{C}_{6} \mathrm{H}_{5} \mathrm{C}_{2} \mathrm{H}_{4}\right.$ $\left.\mathrm{NH}_{3}\right)_{2}-\mathrm{Pbl}_{4}$ perovskite. Opt. Express 18, 5912-5919 (2010).

15. Li, R. et al. Room-temperature electroluminescence from two-dimensional lead halide perovskites. Appl. Phys. Lett. 109, 151101 (2016).

16. Ishihara, T. Optical properties of Pbl-based perovskite structures. J. Lumin. 60-61, 6 (1994).

17. Binek, A., Hanusch, F. C., Docampo, P. \& Bein, T. Stabilization of the trigonal hightemperature phase of formamidinium lead iodide. J. Phys. Chem. Lett. 6, 1249-1253 (2015).

18. Koh, T. M. et al. Formamidinium-containing metal-halide: an alternative material for near-ir absorption perovskite solar cells. J. Phys. Chem. C. 118, 16458-16462 (2014).

19. Cao, D. H., Stoumpos, C. C., Farha, O. K., Hupp, J. T. \& Kanatzidis, M. G. 2D homologous perovskites as light-absorbing materials for solar cell applications. J. Am. Chem. Soc. 137, 7843-7850 (2015).

20. Smith, I. C., Hoke, E. T., Solis-lbarra, D., McGehee, M. D. \& Karunadasa, H. I. A layered hybrid perovskite solar-cell absorber with enhanced moisture stability. Angew. Chem. Int. Ed. 53, 11232-11235 (2014).

21. Quan, L. N. et al. Ligand-stabilized reduced-dimensionality perovskites. J. Am. Chem. Soc. 138, 2649-2655 (2016).
22. Tanaka, K. \& Kondo, T. Bandgap and exciton binding energies in lead-iodidebased natural quantum-well crystals. Sci. Tech. Adv. Mater. 4, 599-604 (2016).

23. $\mathrm{Yu}, \mathrm{J}$. C. et al. Improving the stability and performance of perovskite lightemitting diodes by thermal annealing treatment. Adv. Mater. 28, 6906-6913 (2016).

24. Mello, J. C., Wittmann, H. F. \& Friend, R. H. An improved experimental determination of external photoluminescence quantum efficiency. Adv. Mater. 9, 230-232 (1997).

(i) Open Access This article is licensed under a Creative Commons Attribution 4.0 International License, which permits use, sharing, adaptation, distribution and reproduction in any medium or format, as long as you give appropriate credit to the original author(s) and the source, provide a link to the Creative Commons license, and indicate if changes were made. The images or other third party material in this article are included in the article's Creative Commons license, unless indicated otherwise in a credit line to the material. If material is not included in the article's Creative Commons license and your intended use is not permitted by statutory regulation or exceeds the permitted use, you will need to obtain permission directly from the copyright holder. To view a copy of this license, visit http://creativecommons. org/licenses/by/4.0/.

(c) The Author(s) 2018 